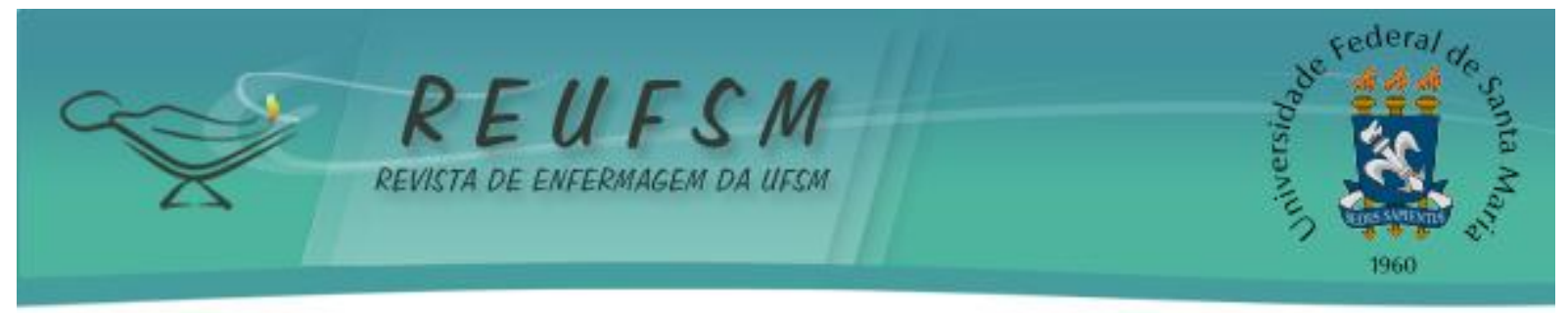

ARTIGO DE REVISÃO

\title{
DEFINIÇÕES CONCEITUAIS E OPERACIONAIS DAS CARACTERÍSTICAS DEFINIDORAS DO DIAGNÓSTICO DE ENFERMAGEM DÉBITO CARDÍACO DIMINUÍDO
}

\section{CONCEPTUAL AND OPERATIONAL DEFINITIONS OF DEFINING CHARACTERISTICS OF DECREASED CARDIAC OUTPUT NURSING DIAGNOSIS}

\author{
DEFINICIONES CONCEPTUALES Y OPERACIONALES DE LAS CARACTERÍSTICAS \\ DEFINIDORAS DEL DIAGNÓSTICO DE ENFERMERÍA GASTO CARDÍACO BAJO
}

Quenia Camille Soares Martins ${ }^{1}$

Patrícia Fernandes Meireles ${ }^{2}$

Eneida Rejane Rabelo ${ }^{3}$ Graziella Badin Aliti ${ }^{4}$

RESUMO: Estudo de atualização por meio de revisão narrativa da literatura. Objetivo: elaborar as definições conceituais e operacionais das 38 características definidoras do diagnóstico de enfermagem Débito Cardíaco Diminuído, apresentadas pela NANDA-I 20092011. Preservando-se o foco de identificação destas características em pacientes com insuficiência cardíaca descompensada. Método: realizada busca de material bibliográfico em livros de semiologia baseada em evidência e artigos na base de dados PUBMED e MEDLINE. Resultado: as definições elaboradas foram analisadas criticamente por três peritas em semiologia cardiovascular e, após avaliação e ajustes, consolidadas tal como apresentadas neste artigo. Conclusão: a relevância deste estudo consiste na incorporação de ferramentas concisas e efetivas acerca dos conceitos relacionados às características definidoras deste diagnóstico, determinando, com isso, a melhora na acurácia e favorecendo o processo de raciocínio clínico diagnóstico.

Descritores: Diagnóstico de enfermagem; Insuficiência cardíaca; Débito cardíaco.

ABSTRACT: Update study through narrative review of the literature that aimed. Objective: to elaborate the conceptual and operation definitions of the 38 defining characteristics of nursing diagnoses decreased cardiac output, presented by the NANDA-I 2009-2011. Preserving the focus of identifying these characteristics in patients with acute decompensate heart failure. Method: was conduct a search of publications in books of semiotics based on evidence and articles in the database PubMed and MEDLINE. Results: the definitions developed were reviewed by three experts in cardiovascular semiology and, after evaluation and adjustments, consolidated as presented in this article.Conclusion: the relevance of this update consists in the incorporation of effective tools and concise about the concepts related to defining characteristics classified as not improved in accuracy and favoring the diagnosis of clinical reasoning.

Descriptors: Nursing diagnosis; Heart failure; Cardiac output.

\footnotetext{
${ }^{1}$ Enfermeira. Doutoranda do Programa de Pós Graduação em Medicina: Ciências Médicas, Universidade Federal do Rio Grande do Sul. Professora Assistente. Faculdade de Ciências de Saúde do Trairí/FACISA. Universidade Federal do Rio Grande do Norte. Email: queniacamille@terra.com.br

${ }^{2}$ Enfermeira. Mestranda, Escola de Enfermagem, Universidade Federal de Pernambuco, Recife, PE, Brasil. Email: patmeireles85@gmail.com

${ }^{3}$ Enfermeira. Doutora em Ciências Biológicas. Professor Adjunto, Escola de Enfermagem, Universidade Federal do Rio Grande do Sul, Porto Alegre, RS, Brasil. E-mail: eneirdarabelo@gmail.com

${ }^{4}$ Enfermeira do Hospital das Clínicas de Porto Alegre, RS, Brasil. Doutoranda, Programa de Pós Graduação Ciências da Saúde: Cardiologia e Ciências Cardiovasculares Universidade Federal do Rio Grande do Sul, Porto Alegre, RS, Brasil. E-mail: graziella.aliti@gmail.com
} 


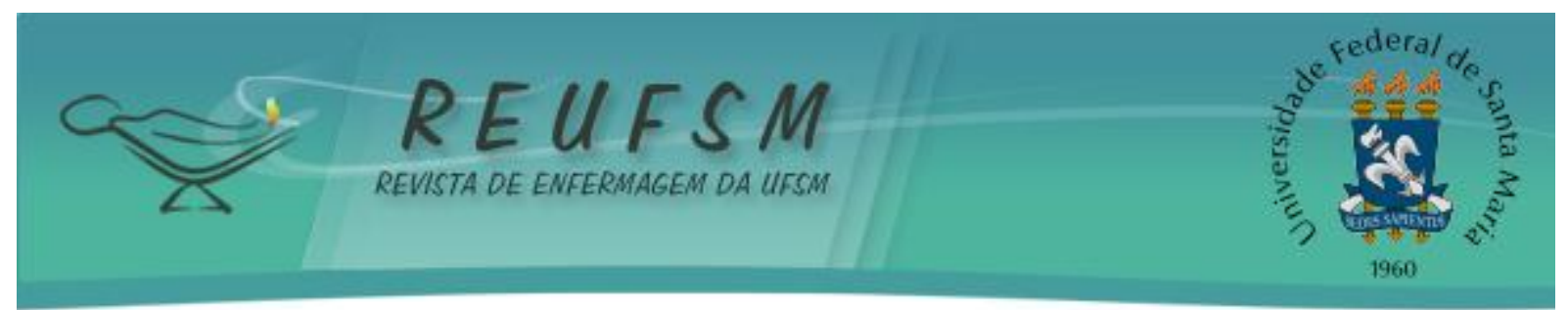

RESUMEN: Estudio de actualización a través de revisión narrativa de la literatura. Objetivo: elaborar las definiciones conceptuales y operacionales de las 38 características definidoras del diagnóstico de enfermería Gasto cardíaco Bajo presentados por NANDA-I 2009-2011.Preservar el enfoque de la identificación de estas características en los pacientes con insuficiencia cardíaca descompensada (ICAD). Método: realizó una búsqueda de las publicaciones en los libros de la semiótica basada en la evidencia y artículos en la base de datos PUBMED y MEDLINE. Resultado: las definiciones desarrolladas fueron revisadas por tres expertos en la sintomatología cardiovascular y después de la evaluación y ajustes, consolidada como se presenta en este artículo. Conclusión: la relevancia de este estudio consiste en la incorporación de herramientas eficaces y sencillas acerca de los conceptos relacionados con características definidoras de diagnóstico para determinar con ello la mejora en la precisión y favoreciendo el diagnóstico del razonamiento clínico. Descriptores: Diagnóstico de enfermería; Insuficencia cardíaca; Gasto cardíaco.

\section{INTRODUÇÃO}

A insuficiência cardíaca (IC) é considerada um dos maiores problemas de saúde pública em todo o mundo, sendo associada a elevados custos e frequentes admissões hospitalares. ${ }^{1}$ Configurando-se como uma síndrome clínica complexa, é caracterizada pela incapacidade do coração de manter um débito cardíaco adequado às necessidades metabólicas dos tecidos. ${ }^{2}$

No ano de 2004, a IC representou a primeira causa de internação hospitalar no Sistema Único de Saúde, em idosos a partir dos 65 anos, sendo que, o aumento da expectativa de vida e a melhora dos padrões terapêuticos medicamentosos, determinaram uma crescente prevalência da doença. Um estudo brasileiro constatou ainda que, destes pacientes internados, até $1 / 3$ voltam a ser readmitidos nos setores de urgência dentro do primeiro ano pós-alta. ${ }^{3}$

A admissão de pacientes com IC descompensada em unidades de emergência exige, por parte da equipe, uma avaliação rápida e eficiente visando condutas baseadas nas melhores evidências disponíveis. Nessa perspectiva, o exame clínico constitui uma ferramenta clássica para o diagnóstico e o manejo do paciente com IC descompensada, pois a anamnese e o exame físico, combinando baixo custo e factibilidade são componentes capazes de individualizar as intervenções mais adequadas. ${ }^{4}$

No contexto da prática da enfermagem, a execução da Sistematização da Assistência de Enfermagem (SAE) provê um guia para o desenvolvimento de pensamentos que direcionam os julgamentos clínicos necessários. Em sua segunda fase, por meio da utilização dos diagnósticos de enfermagem, é possível identificar de forma prática e eficaz, quais são as situações de saúde que as ações da enfermagem podem promover modificações. ${ }^{5}$

Estudos na área dos Diagnósticos de Enfermagem (DE) em cardiologia ressaltam que o diagnóstico de débito cardíaco diminuído é frequentemente encontrado em pacientes com doenças cardiovasculares, especialmente em pacientes com IC. ${ }^{6-7}$ Este diagnóstico é definido pela taxonomia NANDA-I como quantidade insuficiente de sangue bombeado pelo coração para atender às demandas metabólicas corporais. ${ }^{5}$

$\mathrm{Na}$ literatura, estudos têm evidenciado que a presença de determinados sinais e sintomas podem ser úteis para estratificar os pacientes em níveis de gravidade, risco, hospitalização e mortalidade. ${ }^{8-9} \mathrm{Na}$ enfermagem, à medida que se investigam dados e informações, identificam-se os sinais e sintomas que compõe as características definidoras (CD) dos diagnósticos de enfermagem. Estas funcionam como indícios que são agrupadas como manifestação de uma doença ou estado de saúde. ${ }^{5}$ 


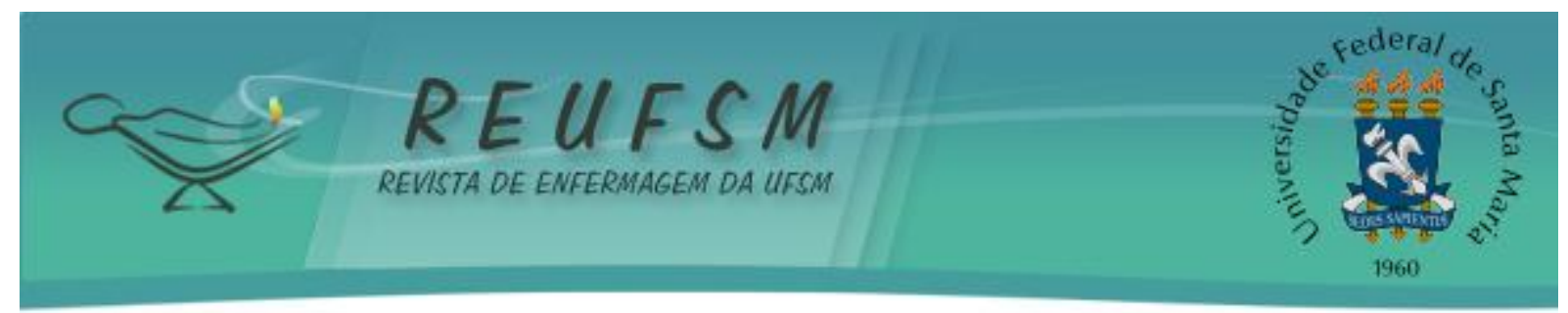

No cenário da SAE, observa-se que nem sempre os pacientes apresentam evidências clínicas que comportem todas as CD para compor um determinado diagnóstico, conforme indicações em livros-textos ou nas classificações que tratam dos diagnósticos de enfermagem. Somado a isso, diferentes diagnósticos compartilham as mesmas $C D$, dificultando seu estabelecimento com alto grau de acurácia. ${ }^{10-11}$ Para sustentar a escolha adequada do diagnóstico de enfermagem em cada situação clínica, é necessário que as bases para essa decisão estejam fortemente ligadas às $C D .^{5}$

Neste contexto, as definições operacionais são componentes vitais nas pesquisas referentes aos diagnósticos de enfermagem, mais especificamente as investigações voltadas às $C D$, pois estabelecem uma ligação entre a observação e a investigação científica. A finalidade dessas definições é descrever o que será mensurado e de que forma pode ser avaliada a presença de um sinal ou de um sintoma. ${ }^{12}$ Além disso, podem servir para aumentar a confiabilidade e a validade dos dados clínicos referentes aos diagnósticos de enfermagem e, portanto, favorecer a replicação de pesquisas, a melhora da habilidade do pesquisador de correlacionar o achado de estudos anteriores e favorecer a construção de indicadores de avaliação das intervenções de enfermagem. ${ }^{13}$

Deste modo, objetivou-se com este estudo, descrever os dados contemporâneos acerca das CD do diagnóstico de enfermagem Débito Cardíaco Diminuído com vistas na elaboração de definições conceituais e operacionais para as 38 CDs contempladas no livro de definições e classificação da NANDA-I 2009-2011. Ressalta-se que foi preservado o foco de identificação das respectivas CD em pacientes com IC descompensada.

\section{MÉTODO}

Realizou-se um estudo de atualização por meio de revisão narrativa da literatura, com vistas na elaboração das definições conceituais e operacionais das CD do diagnóstico de enfermagem Débito Cardíaco Diminuído. Esta foi embasada na busca de material bibliográfico formado por livros de semiologia baseada em evidência, publicados entre 2000 2011 e artigos originais na base de dados PUBMED e MEDLINE, no período de 1989 a 2011. Para esta busca foram utilizados os seguintes descritores: diagnóstico de enfermagem (nursing diagnosis), débito cardíaco (cardiac output) e insuficiência cardíaca (heart failure), sendo a busca realizada nos idiomas português e inglês. Foram incluídos artigos disponíveis em sua integralidade nas bases de dados selecionadas, artigos disponíveis em português, inglês ou espanhol que abordavam aspectos da semiologia e semiotécnica em pacientes adultos (idade superior a 18 anos), portadores de IC e aqueles relativos ao diagnóstico débito cardíaco diminuído. Foram excluídos artigos cujas bases propedêuticas eram generalistas ou não abordavam a temática relevante ao alcance do objetivo do estudo, além de editoriais, cartas ao editor e artigos que não eram acessíveis na íntegra.

Alguns marcos teóricos foram utilizados para evidenciar a necessidade de atualização acerca desta temática. ${ }^{4,8}$ Posteriormente, essas características, bem como suas definições, foram avaliadas criticamente por três peritas na área de semiologia cardiovascular. Para a escolha das peritas foram utilizados critérios adaptados do modelo de Fhering ${ }^{14}$ para validação de diagnósticos de enfermagem no qual foram pontuados os seguintes itens: doutorado em ciências cardiovasculares, mestrado em ciências cardiovasculares, artigos publicados sobre IC em revistas científicas, artigos publicados sobre SAE em revistas científicas, prática clínica mínima de cinco anos em linguagem da SAE, prática atual mínima de cinco anos na área de interesse, título de especialista em enfermagem cardiovascular, experiência docente em semiologia cardiovascular. Com bases, nos itens descritos, foi necessária obtenção de pontuação igual ou superior a cinco para que fossem consideradas peritas. No presente estudo estas obtiveram pontuação superior a 16 pontos. 


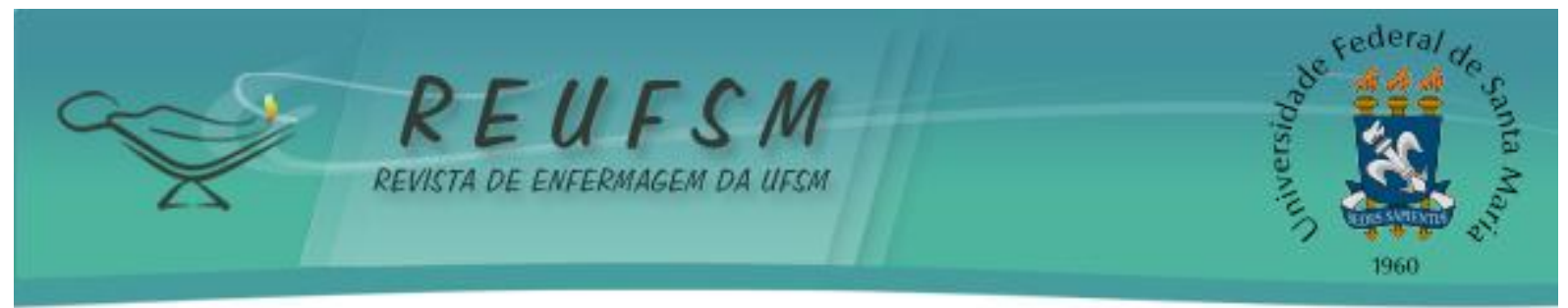

Após a elaboração das definições e da realização de consenso pelos pareceres destes enfermeiros, as definições foram consolidadas.

\section{RESULTADOS}

A consolidação das definições conceituais e operacionais para as CD do diagnóstico de enfermagem Débito Cardíaco Diminuído encontra-se descrita no quadro abaixo.

\begin{tabular}{|c|c|}
\hline \begin{tabular}{l}
\multicolumn{2}{l}{ Características } \\
definidoras do DE \\
Débito \\
Diminuído
\end{tabular} & Definição conceitual/operacional \\
\hline $\begin{array}{l}\text { Distensão da veia } \\
\text { jugular }\end{array}$ & $\begin{array}{l}\text { Medida que indica a alteração de volume das câmaras cardíacas } \\
\text { direitas. Deve ser avaliada com o paciente em decúbito dorsal a } 45^{\circ} .^{12} \\
\text { Em pacientes congestos, a turgência da veia jugular pode estar } \\
\text { presente no limite a mais do que três ou quatro cm acima do ângulo } \\
\text { esternal. }{ }^{15}\end{array}$ \\
\hline Edema & $\begin{array}{l}\text { É definido como o acúmulo de líquidos no espaço intersticial como } \\
\text { consequência de alterações na homeostase do sódio e da água. } 0 \\
\text { edema pode ser generalizado ou localizado. Sua avaliação pode ser } \\
\text { realizada pela verificação do aumento de peso ou através do sinal de } \\
\text { cacifo ou de Godet, ou seja, pela compressão da região pré-tibial com o } \\
\text { polegar, por cerca de } 10 \text { segundos e observando se há a formação de } \\
\text { depressão. A profundidade da depressão pode variar de } 0 / 4 \text { a } 4 / 4 \text {, em } \\
\text { uma escala de cruzes }(-/ 4+,+/ 4,++/ 4+,++/ 4 \text { e }++++/ 4) .{ }^{16-17}\end{array}$ \\
\hline $\begin{array}{lr}\text { Pressão } & \text { Venosa } \\
\text { Central } & \text { (PVC) } \\
\text { aumentada/diminuída }\end{array}$ & $\begin{array}{l}\text { A PVC é utilizada para avaliar a função ventricular direita e o retorno } \\
\text { venoso para as câmaras cardíacas direitas. Os valores normais situam-se } \\
\text { entre oito e } 12 \mathrm{~cm} \text { de } \mathrm{H}_{2} \mathrm{O} \text { quando medidos por meio de coluna d'água. }{ }^{18} \\
\text { Pela inspeção, também é possível fazer uma estimativa da PVC. Para } \\
\text { tanto, localiza-se o menisco da coluna líquida da veia jugular externa e } \\
\text { traça-se uma linha horizontal até o manúbrio esternal. O átrio direito } \\
\text { localiza-se a cinco cm do manúbrio. Logo, a distância entre o manúbrio } \\
\text { e a linha imaginária traçada a partir do menisco somada a cinco-fornece } \\
\text { a pressão atrial aproximada em centímetros de água. }{ }^{19}\end{array}$ \\
\hline Ganho de peso & $\begin{array}{l}\text { É o aumento do peso corporal em até } 1,3 \mathrm{Kg} \text { em dois dias ou de } 1,3 \text { a } \\
2,2 \mathrm{Kg} \text { em uma semana. Essa variação de peso pode ser um indicativo } \\
\text { de que existe retenção de líquidos e isso se torna um fator preditivo de } \\
\text { piora da IC. A verificação do peso deve ocorrer pela manhã após urinar, } \\
\text { com roupas leves, antes do café e utilizando a mesma balança. }{ }^{20-21}\end{array}$ \\
\hline
\end{tabular}




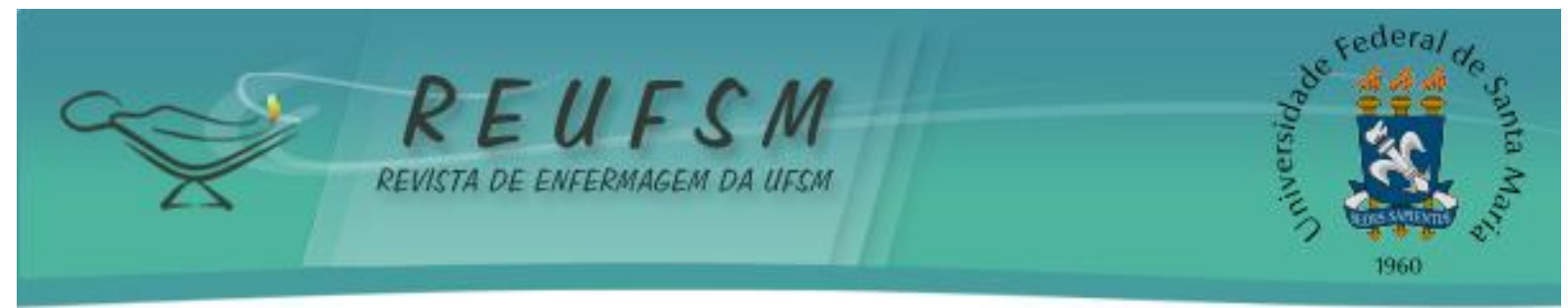

\begin{tabular}{|c|c|}
\hline Dispnéia & $\begin{array}{l}\text { E o termo utilizado para caracterizar uma experiência subjetiva de } \\
\text { desconforto respiratório que está se compondo de sensações } \\
\text { qualitativamente distintas, podendo variar de intensidade. }{ }^{22} \text { Essa } \\
\text { sensação ocorre secundariamente à redução da perfusão cardíaca e } \\
\text { pulmonar - consequência da redução da contratilidade cardíaca. A } \\
\text { dispnéia em geral é resolvida ou melhorada com a administração de } \\
\text { oxigênio complementar, com a utilização de diuréticos ou com a } \\
\text { redução da pré-carga. A intensidade da dispnéia pode ser avaliada por } \\
\text { fatores cognitivos e contextuais. }{ }^{23-24} \text { Pode ser identificada por meio de } \\
\text { alterações da frequência respiratória, estridor, agitação, utilização de } \\
\text { musculatura acessória para os movimentos respiratórios e alterações na } \\
\text { ausculta cardíaca e pulmonar. }{ }^{25}\end{array}$ \\
\hline Oligúria & $\begin{array}{l}\dot{E} \text { o débito urinário diminuído relacionado à perfusão renal reduzida } \\
\text { pela queda do débito cardíaco. Geralmente ocorre devido à redução do } \\
\text { fluxo sanguíneo renal ou por lesões renais. Esse achado deve ser } \\
\text { mensurado por meio da medida do volume de diurese. A oligúria se } \\
\text { caracteriza por diurese inferior a } 400 \mathrm{~mL} / 24 \mathrm{~h} \text { ou menos de } 20 \mathrm{~mL} / \mathrm{h} .{ }^{26}\end{array}$ \\
\hline $\begin{array}{l}\text { Variações nas leituras } \\
\text { de pressão arterial } \\
\text { (hipotensão } \\
\text { ortostática) }\end{array}$ & $\begin{array}{l}\text { Na IC, a hipotensão ortostática é um achado comum. }{ }^{29} \bar{E} \text { a diminuição da } \\
\text { pressão sistólica em função da redução da força de contração do ventrículo } \\
\text { esquerdo e aumento da pressão diastólica relacionada à hiperatividade } \\
\text { adrenérgica. A hipotensão postural (ortostática) é definida como a queda de } \\
\text { pressão de } 20 \mathrm{mmHg} \text { na pressão sistólica ou } 10 \mathrm{mmHg} \text { na diastólica com a } \\
\text { troca da posição deitada para a ortostática. }{ }^{15-27} \mathrm{~A} \text { aferição da pressão arterial } \\
\text { é realizada, preferencialmente, nos membros superiores, utilizando-se o } \\
\text { esfigmomanômetro e o estetoscópio biauricular. As médias anteriores de } \\
\text { pressão arterial e as informações do paciente podem ser úteis para avaliar } \\
\text { possíveis desvios da normalidade. }{ }^{12}\end{array}$ \\
\hline Agitação & $\begin{array}{l}\text { É a alteração do comportamento com irritabilidade, mau humor, } \\
\text { instabilidade psicomotora e inquietação, sendo observadas pelo } \\
\text { examinador e validadas por informações de familiares e do paciente. } \\
\text { Para investigação desse achado, deve-se avaliar o estado atual do } \\
\text { paciente, buscando informações a cerca de sua história recente. }{ }^{12}\end{array}$ \\
\hline Ansiedade & $\begin{array}{l}\text { É o sentimento incômodo de desconforto ou temor, seguido por resposta } \\
\text { autonômica (a fonte é frequentemente não específica ou desconhecida } \\
\text { para o indivíduo). Reflete a apreensão causada pela antecipação do } \\
\text { perigo; um sinal de alerta que remete perigo iminente levando o }\end{array}$ \\
\hline
\end{tabular}




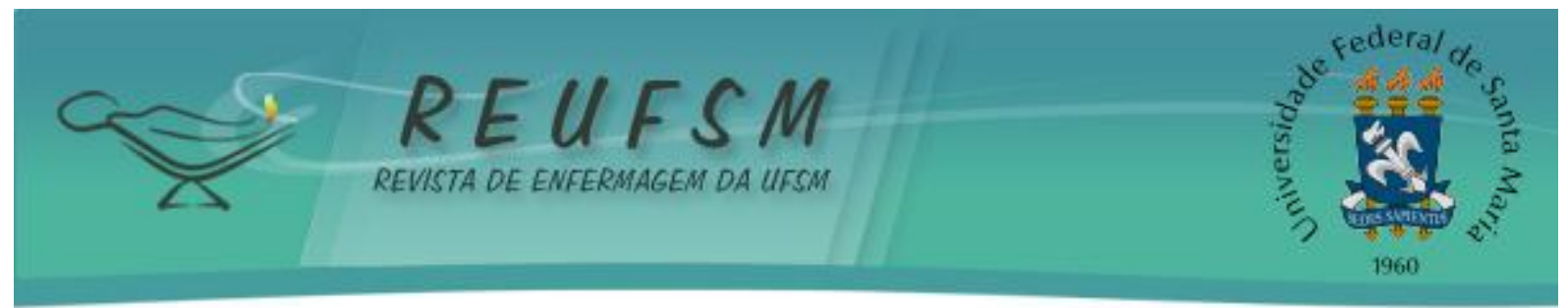

\begin{tabular}{|c|c|}
\hline & $\begin{array}{l}\text { indivíduo a implementar medidas para lidar com a ameaça. }{ }^{5} \text { Pode ser } \\
\text { identificada durante a entrevista. }{ }^{28}\end{array}$ \\
\hline $\begin{array}{l}\text { Som cardíaco de } \\
\text { terceira bulha (B3) }\end{array}$ & $\begin{array}{l}\text { Consiste na ausculta cardíaca de um ruído "adicional" que ocorre no } \\
\text { início da diástole e é mais audível com o paciente deitado em decúbito } \\
\text { lateral esquerdo. É auscultada como um galope, causado pelo } \\
\text { cadenciamento das três bulhas. }{ }^{29} \text { É auscultado em região apical com o } \\
\text { uso da campânula. O uso do decúbito lateral esquerdo facilita sua } \\
\text { identificação. }{ }^{19}\end{array}$ \\
\hline Crepitações & $\begin{array}{l}\text { Consistem em ruídos pulmonares adventícios ouvidos no final da } \\
\text { inspiração. Têm alta frequência e são causados por presença de líquido } \\
\text { nos alvéolos e bronquíolos terminais. São evidenciados, inicialmente, } \\
\text { nas bases, podendo progredir para as outras áreas pulmonares. Este é } \\
\text { um achado que pode ser obtido por meio da técnica de ausculta } \\
\text { pulmonar. }\end{array}$ \\
\hline $\begin{array}{l}\text { Dispnéia paroxística } \\
\text { noturna }\end{array}$ & $\begin{array}{l}\text { É definida como a situação na qual o indivíduo tem seu sono interrompido } \\
\text { por uma sensação importante de falta de ar, levando o paciente a sentar } \\
\text { no leito. Este é um dado obtido através da entrevista com o paciente, em } \\
\text { busca de questões sobre o sono. }^{22}\end{array}$ \\
\hline Ortopnéia & $\begin{array}{l}\text { É a dificuldade respiratória que aparece ou agrava com a adoção da } \\
\text { posição horizontal, tendendo a ser aliviada, parcial ou totalmente, com } \\
\text { a elevação da parte superior do tórax pelo uso de um número maior de } \\
\text { travesseiros. }{ }^{25} \text { Pode ser identificada por meio da anamnese e/ou exame } \\
\text { físico. }{ }^{31}\end{array}$ \\
\hline $\begin{array}{l}\text { Fração de ejeção } \\
\text { diminuída }\end{array}$ & $\begin{array}{l}\text { A fração de ejeção quantifica a função sistólica ventricular global e } \\
\text { pode ser definida como a fração de sangue ejetada a cada contração } \\
\text { ventricular. A fração de ejeção normal é de aproximadamente } 60 \% \text { e } \\
\text { pode ser quantificada por métodos invasivos (ventriculografia) e não } \\
\text { invasivos (ecocardiograma). }\end{array}$ \\
\hline $\begin{array}{l}\text { Índice } \\
\text { diminuído }\end{array}$ & $\begin{array}{l}0 \text { índice cardíaco pode ser definido por meio da divisão do débito } \\
\text { cardíaco pela superfície corporal. Seus parâmetros de normalidade } \\
\text { encontram-se entre } 2,5 \text { e } 4 \mathrm{~L} / \mathrm{min} / \mathrm{m} .{ }^{2,33} \text { Essa medida pode ser obtida à } \\
\text { beira do leito com o método de termodiluição por meio da utilização do } \\
\text { cateter de Swan-Ganz. Pode ser verificado também por meio de } \\
\text { ecocardiograma e/ ou cateterismo cardíaco direito. }{ }^{34}\end{array}$ \\
\hline $\begin{array}{lr}\begin{array}{l}\text { Índice do trabalho } \\
\text { sistólico }\end{array} & \text { do }\end{array}$ & $\begin{array}{l}\text { É o trabalho executado pelo ventrículo para ejetar o índice do volume } \\
\text { sistólico por meio de um gradiente pressórico na aorta. Esse indicador é }\end{array}$ \\
\hline
\end{tabular}




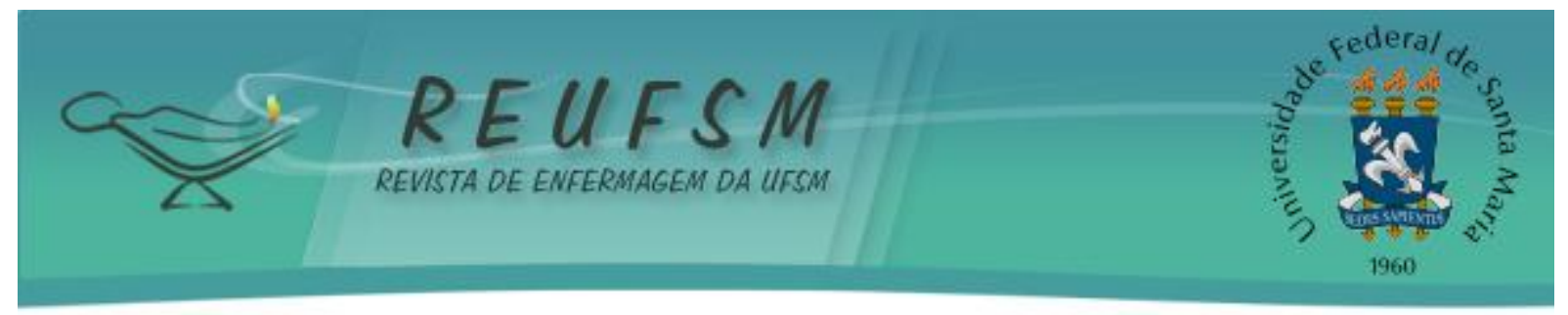

\begin{tabular}{|c|c|}
\hline $\begin{array}{l}\text { ventrículo esquerdo } \\
\text { diminuído }\end{array}$ & $\begin{array}{l}\text { obtido através de aferição por meio do cateter de Swan-Ganz. Para sua } \\
\text { obtenção, realiza-se um cálculo utilizando a fórmula (pressão arterial } \\
\text { média - pressão de oclusão da artéria pulmonar) } \times \text { índice do volume } \\
\text { sistólico } \times 0,0136 \text {. Os valores normais situam-se entre } 50-80 \mathrm{~g} / \mathrm{m}^{2} / \text { bat. }^{34}\end{array}$ \\
\hline $\begin{array}{l}\text { Índice do volume } \\
\text { sistólico diminuído }\end{array}$ & $\begin{array}{l}\text { Também é conhecido como índice sistólico e pode ser calculado com } \\
\text { relação à massa corpórea. A monitorização hemodinâmica com o } \\
\text { cateter de artéria pulmonar (Swan-Ganz) fornece esse parâmetro. Este } \\
\text { é obtido por meio da fórmula volume sistólico/massa corpórea ou índice } \\
\text { cardíaco/frequência cardíaca. Valores de referência 30-60 } \\
\mathrm{mL} / \text { bat/min. }{ }^{34}\end{array}$ \\
\hline $\begin{array}{l}\text { Resistência vascular } \\
\text { pulmonar } \\
\text { aumentada/diminuída }\end{array}$ & $\begin{array}{l}\text { A resistência vascular pulmonar (RVP) reflete a medida de tônus do } \\
\text { sistema vascular pulmonar. Esse parâmetro pode ser obtido por meio do } \\
\text { cateter de Swan-Ganz. O cálculo da RVP baseia-se na fórmula (pressão } \\
\text { da artéria pulmonar - pressão de oclusão da artéria pulmonar) × } 80 \text { / } \\
\text { débito cardíaco. Os valores normais variam de } 20-120 \text { dina-s/ } \mathrm{cm}^{-5} \cdot{ }^{33-34}\end{array}$ \\
\hline $\begin{array}{l}\text { Resistência vascular } \\
\text { sistêmica } \\
\text { aumentada/diminuída }\end{array}$ & $\begin{array}{l}\text { A resistência vascular sistêmica é uma medida do tônus vascular total. } \\
\text { Essa medida pode ser obtida por meio do método de termo diluição } \\
\text { utilizando-se o cateter de Swan-Ganz. Pode ser obtida através do } \\
\text { cálculo da seguinte fórmula: (pressão arterial média - pressão venosa } \\
\text { central) / índice cardíaco. Os valores normais para a resistência } \\
\text { vascular sistêmica são entre } 1600 \text { e } 2400 \text { dina-s/cm }{ }^{-5} \cdot 34\end{array}$ \\
\hline $\begin{array}{l}\text { Som cardíaco quarta } \\
\text { bulha (B4) }\end{array}$ & $\begin{array}{l}\text { Este é um som cardíaco avaliado por meio de técnica de ausculta } \\
\text { cardíaca. A quarta bulha (B4) é um som de baixa frequência, pré- } \\
\text { sistólico, correspondente à sístole atrial frente a um ventrículo com } \\
\text { alteração no relaxamento. Deve ser auscultado na região apical, } \\
\text { utilizando a campânula, logo antes da primeira bulha. }{ }^{19}\end{array}$ \\
\hline $\begin{array}{l}\text { Alterações no } \\
\text { eletrocardiograma }\end{array}$ & $\begin{array}{l}\text { Algumas alterações comuns em pacientes com IC são: sobrecarga de } \\
\text { câmeras cardíacas; bloqueios intraventriculares; arritmias supra ou } \\
\text { ventriculares. Essas alterações podem ser verificadas através das } \\
\text { leituras obtidas por meio do eletrocardiograma. }{ }^{35}\end{array}$ \\
\hline & $\begin{array}{l}\text { Alteração na geração e/ou condução do impulso elétrico. Extra-sístoles } \\
\text { atriais: descargas precoces geradas em um foco ectópico, causando uma } \\
\text { onda P anormal ao eletrocardiograma. Quando ocorrem precocemente na } \\
\text { onda T do batimento precedente, podem induzir a fibrilação atrial. } \\
\text { Fibrilação atrial: ocorre quando as fibras musculares se contraem } \\
\text { independentemente. Quando isso acontece nas fibras do músculo atrial, }\end{array}$ \\
\hline
\end{tabular}




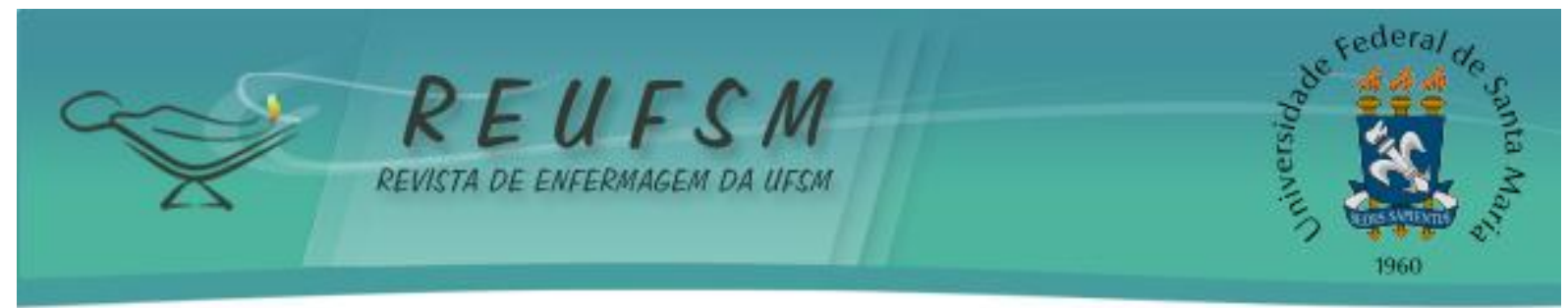

\begin{tabular}{|c|c|}
\hline & $\begin{array}{l}\text { não existem ondas P no eletrocardiograma; há apenas uma linha irregular. } \\
\text { Taquicardia ventricular: ocorre quando um foco no músculo ventricular } \\
\text { despolariza com uma frequência maior. Assim, esse estímulo se propaga } \\
\text { por uma via anormal, através da musculatura atrial, e o complexo QRS } \\
\text { torna-se alargado e anormal. As arritmias podem ser observadas por meio } \\
\text { da monitorização cardíaca ou por meio do eletrocardiograma. }{ }^{35}\end{array}$ \\
\hline Palpitações & $\begin{array}{l}\text { A palpitação é definida como a percepção desagradável de batimentos } \\
\text { cardíacos vigorosos ou rápidos, podendo associar-se a quadros } \\
\text { respiratórios ou cardiovasculares; além disso, associa-se em diferentes } \\
\text { graus à intolerância à atividade física. Esta deve ser avaliada por meio } \\
\text { de entrevista. }{ }^{15}\end{array}$ \\
\hline Taquicardia & $\begin{array}{l}\text { A taquicardia é definida como frequência cardíaca acima de } 100 \\
\text { batimentos por minuto. Esta deve ser avaliada no início do exame físico ou } \\
\text { junto com o exame cardiovascular. Costumam-se utilizar os pulsos radial, } \\
\text { braquial ou carotídeo para tal verificação. } 0 \text { avaliador deve ficar em frente } \\
\text { ao paciente e comprimir a artéria escolhida com o dedo indicador e médio; } \\
\text { depois, deve-se proceder a contagem dos batimentos cardíacos por um } \\
\text { minuto. }^{27}\end{array}$ \\
\hline Bradicardia & $\begin{array}{l}\text { A bradicardia é definida como frequência cardíaca inferior a } 60 \\
\text { batimentos por minuto. A frequência cardíaca deve ser avaliada no } \\
\text { início do exame físico ou junto com o exame cardiovascular. } \\
\text { Rotineiramente, utilizam-se os pulsos radial, braquial ou carotídeo para } \\
\text { tal verificação. O avaliador deve ficar em frente ao paciente e } \\
\text { comprimir a artéria escolhida com o dedo indicador e médio; depois } \\
\text { disso, deve-se proceder a contagem dos batimentos cardíacos por um } \\
\text { minuto. }{ }^{27}\end{array}$ \\
\hline Fadiga & $\begin{array}{l}\text { Sensação opressiva apoiada por exaustão e capacidade reduzida para a } \\
\text { realização de atividades físicas e mentais em níveis habituais. }{ }^{5} \text { Esta é } \\
\text { uma condição associada com a redução das atividades físicas diárias ou } \\
\text { diminuição da condição funcional como mensurado pela classificação da } \\
\text { New York Heart Association (NYHA). }{ }^{36} \text { Essa mensuração ocorre através } \\
\text { da estratificação dos pacientes em classes funcionais: } \\
\text { Classe funcional I - Nenhuma limitação física em atividades rotineiras. } \\
\text { Classe funcional II - Leves sintomas e limitações ligeiras em atividades } \\
\text { rotineiras. Confortável em repouso. } \\
\text { Classe funcional III - Limitação importante da atividade física; }\end{array}$ \\
\hline
\end{tabular}




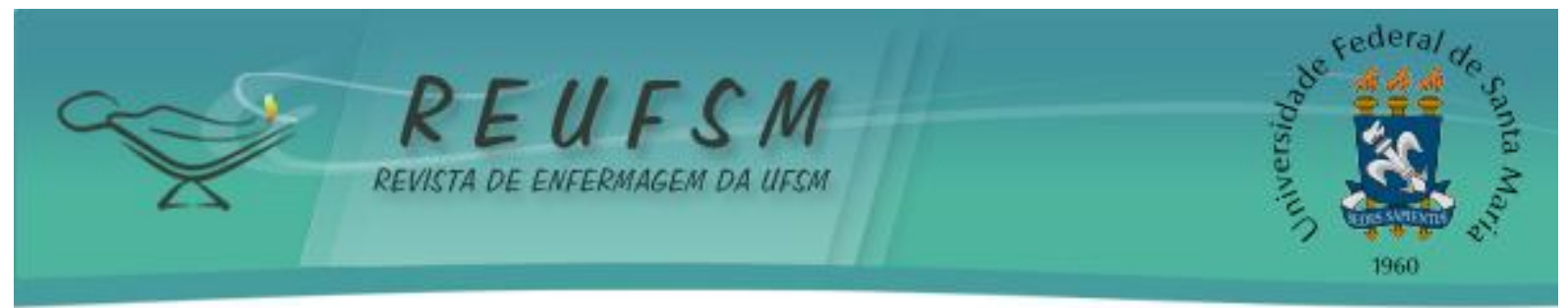

\begin{tabular}{|c|c|}
\hline & 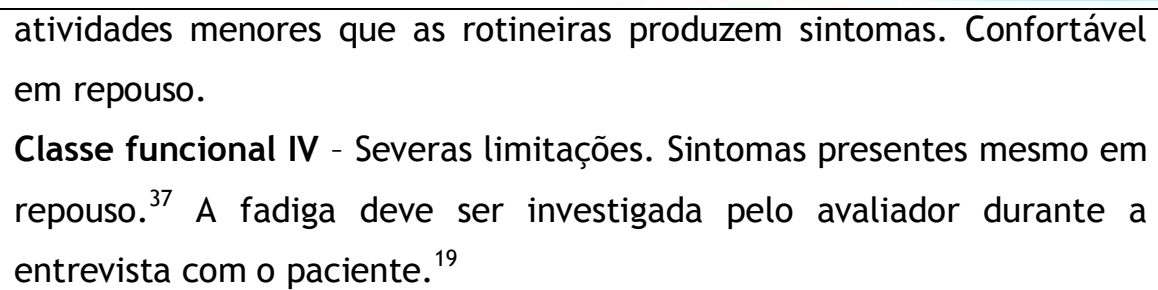 \\
\hline $\begin{array}{l}\text { Débito } \\
\text { diminuído }\end{array}$ & $\begin{array}{l}\text { É definido como a quantidade de sangue ejetada pelo coração por } \\
\text { minuto. Pode ser avaliado à beira do leito utilizando cateter de Swan- } \\
\text { Ganz, ou verificado por meio de cateterismo cardíaco direito ou } \\
\text { ecocardiograma. }{ }^{33} \text { Essa medida pode ser obtida através da fórmula DC = } \\
\text { quantidade de sangue ejetado a cada contração ventricular } \times \\
\text { frequência cardíaca. Os valores normais variam entre } 4 \text { e } 5 \text { L/min. }{ }^{33} \\
\text { Uma outra forma utilizada para estimar o baixo débito é a verificação } \\
\text { da pressão proporcional de pulso (PPP); costuma ser avaliada em forma } \\
\text { de índice por meio da seguinte fórmula: (PAS - PAD) / PAS, onde PAS é a } \\
\text { pressão arterial sistólica e PAD, a pressão arterial diastólica. } \\
\text { Consideram-se os valores de PPP inferiores a 0,25 como preditores de } \\
\text { baixo débito cardíaco. }{ }^{8}\end{array}$ \\
\hline $\begin{array}{l}\text { Pressão pulmonar } \\
\text { capilar } \\
\text { aumentada/diminuída }\end{array}$ & $\begin{array}{l}\dot{E} \text { a medida equivalente à pressão de enchimento ventricular e é } \\
\text { frequentemente empregada como medida substituta da pré-carga. Esta é uma } \\
\text { medida obtida por meio do cateter de Swan-Ganz. Os valores de normalidade } \\
\text { situam-se menores ou iguais a } 12 \mathrm{mmHg} .{ }^{33}\end{array}$ \\
\hline Pele fria e pegajosa & $\begin{array}{l}\text { A pele fria e pegajosa reflete a vasoconstrição periférica, causando } \\
\text { redução da temperatura cutânea, acompanhada de sudorese. Ambos } \\
\text { são sinais de hiperatividade adrenérgica compensatória da redução de } \\
\text { contratilidade e débito cardíaco. }{ }^{31} 0 \text { exame da pele compreende a } \\
\text { inspeção das alterações cutâneas e, após sua palpação, que subsidia } \\
\text { informações sobre a textura e demais aspectos. }{ }^{38}\end{array}$ \\
\hline \multirow[t]{2}{*}{$\begin{array}{l}\text { Reperfusão capilar } \\
\text { periférica prolongada }\end{array}$} & $\begin{array}{l}\text { Esta é uma condição em que o leito ungueal torna-se esbranquiçado, } \\
\text { retornando à coloração normal em período prolongado. A reperfusão } \\
\text { capilar periférica é avaliada pela compressão da polpa de um ou mais } \\
\text { dígitos, e liberando-se posteriormente. } 0 \text { tempo gasto para isso equivale à } \\
\text { reperfusão capilar periférica, e normalmente, não ultrapassa três } \\
\text { segundos. }^{31}\end{array}$ \\
\hline & $\begin{array}{l}\text { São determinados por uma complexa interação do coração e sistema } \\
\text { arterial e venoso. Os pulsos periféricos mostram-se de difícil palpação, } \\
\text { exercem baixa pressão sobre os dedos e são ocluídos com facilidade }\end{array}$ \\
\hline
\end{tabular}




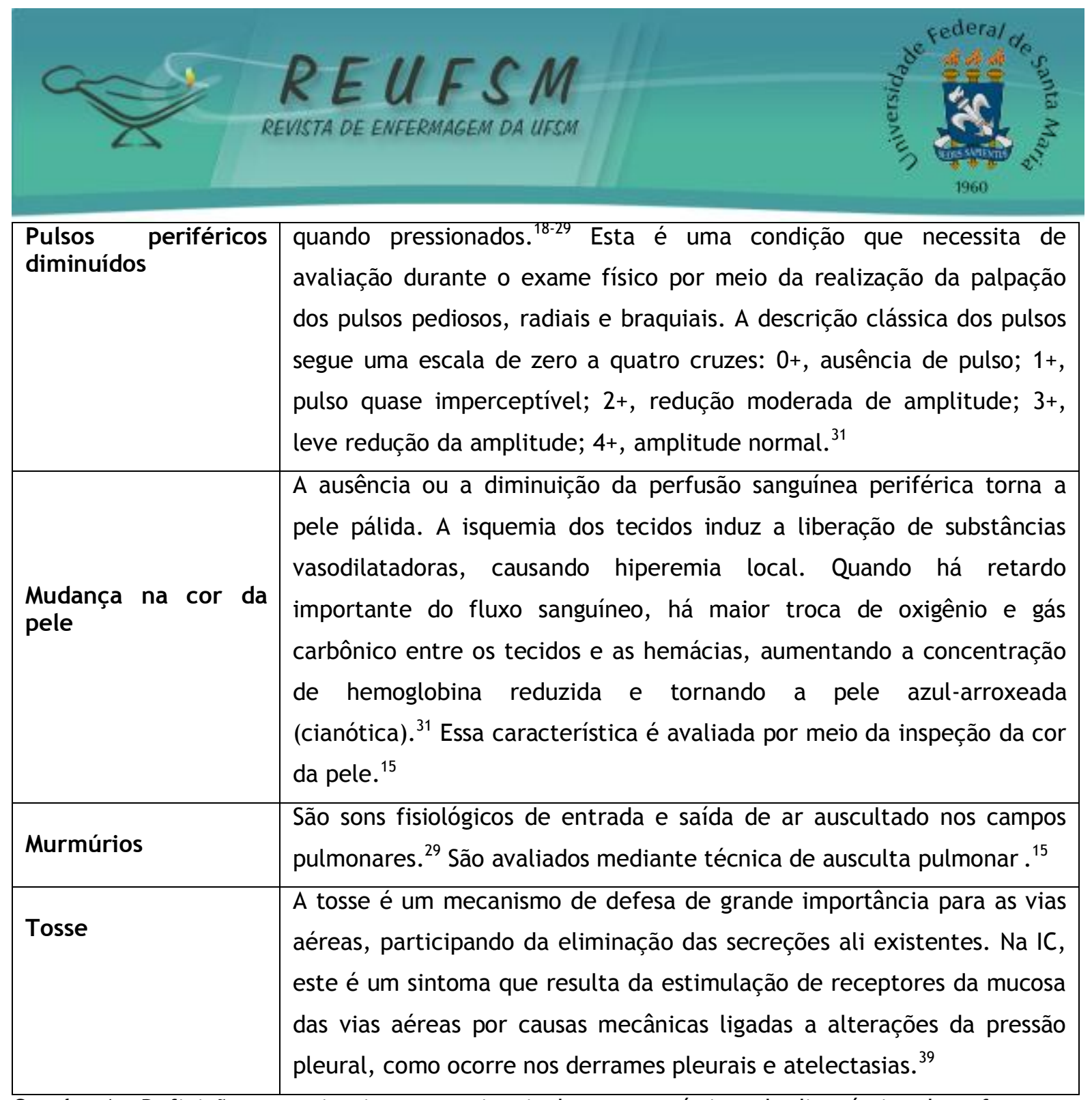

Quadro 1 - Definições conceituais e operacionais das características do diagnóstico de enfermagem Débito Cardíaco Diminuído consolidadas.

\section{DISCUSSÃO}

$\mathrm{Na}$ prática clínica do enfermeiro pode ser difícil identificar as CDs mais fortemente associadas ao débito cardíaco diminuído em pacientes com IC. Além das muitas particularidades relacionadas à clínica do baixo débito cardíaco, o número de estudos que evidenciam as CDs nessa população é escasso. Contudo, recentemente, alguns estudos vêm buscando demonstrar o comportamento das CDs deste diagnóstico..$^{40-43}$

Uma revisão sistemática publicada recentemente com o objetivo de caracterizar os artigos relacionados ao diagnóstico de enfermagem débito cardíaco diminuído evidenciou que nos 13 artigos selecionados foram identificadas cinquenta CDs. Dessas, as dez com maior frequência foram: frequência/ritmo cardíaco e dispnéia, seguidos da labilidade da pressão arterial e estertores, oligúria/anúria e edema, pele fria e fadiga/fraqueza, diminuição dos pulsos periféricos e diminuição da perfusão periférica. 0 mesmo estudo infere ainda que, em diferentes cenários de atuação, é possível identificar as CDs por meio de ferramentas propedêuticas independente dos recursos tecnológicos disponíveis. ${ }^{40}$

Nesta perspectiva, um estudo de validação de conteúdo, que avaliou 79 CDs encontradas na literatura médica e da enfermagem, observou que 38 foram consideradas válidas. Destas, cinco foram consideradas maiores ou principais: débito cardíaco diminuído, 


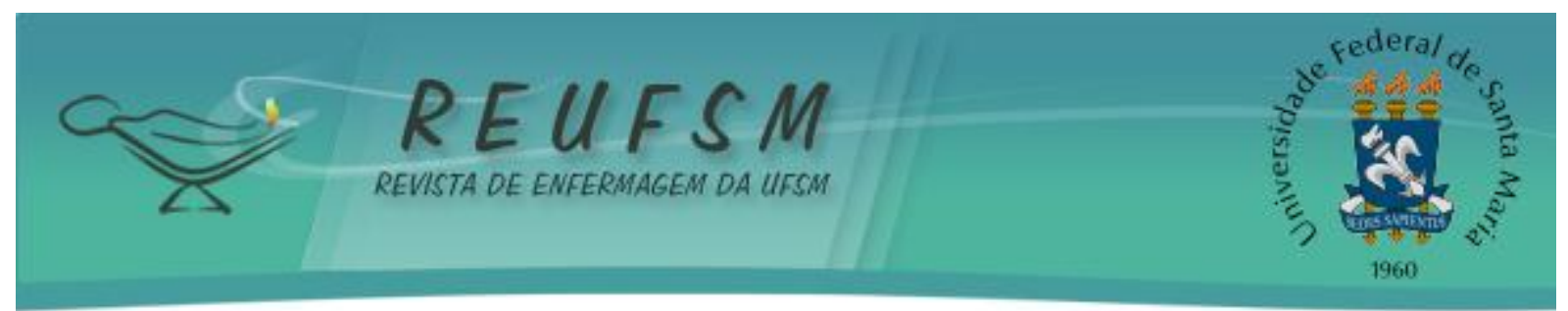

índice cardíaco diminuído, pulso periférico diminuído, aumento do nível sérico de lactato, diminuição da saturação venosa de oxigênio e perfusão capilar periférica diminuída. Observou-se ainda que das 38 CDs validadas apenas 17 encontram-se descritas na NANDA-I. ${ }^{41}$

Neste cenário, cabe ressaltar que diferente do proposto neste estudo, algumas pesquisas ${ }^{40-42}$ sugerem a necessidade de avaliação de outras CD descritas na literatura médica, as quais ainda não estão contempladas pela NANDA-I.

Atualmente existe uma tendência na investigação das CDs por meio da utilização de abordagens não invasivas para evidenciar o débito cardíaco. Uma vez que, a medida comumente utilizada para mensurar débito cardíaco, o cateter arterial pulmonar, além de ser um método extremamente invasivo, pode ainda não fornecer dados suficientes para orientar a prática clínica. ${ }^{42}$ Desse modo, os enfermeiros vêm aperfeiçoando de forma contínua as técnicas de anamnese e exame clínico como forma de instrumentalizarem-se para detectar as CDs para este diagnóstico em pacientes com IC, reduzindo assim a necessidade de recursos tecnológicos para mensurar tais variáveis.

No presente estudo, algumas CDs foram descritas seguindo essa perspectiva. Nas características de pressão venosa central e débito cardíaco são oferecidas ao leitor novas possibilidades propedêuticas para a identificação destas características sem a utilização de métodos invasivos, como a medida estimada da PVC pela distensão venosa jugular ${ }^{19-25}$ e a estimativa da medida de débito cardíaco através da pressão proporcional de pulso. ${ }^{8}$

\section{CONCLUSÕES}

O conhecimento científico embasado e crítico em relação às definições conceituais e operacionais das CD determinam a acurácia no estabelecimento dos DE. Estas definições estabelecem uma ligação entre a observação e a investigação científica, favorecendo a habilidade do enfermeiro em reconhecer e identificar sinais e sintomas que constituem um diagnóstico específico.

Desta forma, a relevância deste estudo consiste na incorporação de ferramentas concisas e efetivas acerca dos conceitos relacionados às CDs deste diagnóstico. No contexto da IC descompensada, este conhecimento constitui-se também em elemento fundamental para o estabelecimento de diagnósticos acurados, favorecendo o processo de raciocínio clínico no diagnóstico dos enfermeiros e, principalmente, o estabelecimento de intervenções seguras e eficientes na admissão de pacientes com esta síndrome.

\section{LIMITAÇÕES DO ESTUDO}

Apesar da incontestável importância do fundamento teórico como fomento para a prática clínica da enfermagem e execução precisa da SAE, são limitados os estudos que contemplem ou subsidiem as definições das CDs relacionadas especificamente aos diagnósticos de enfermagem para o paciente com IC descompensada.

\section{REFERÊNCIAS}

1. Tavares LR, Victer H, Linhares JM, Barros CM, Oliveira MV, Pacheco LC, et al. Epidemiologia da insuficiência cardíaca descompensada em Niterói - Projeto EPICA - Niterói. Arq bras cardiol. 2004;82(2):121-4.

2. Silva Neto LB, Clausell N. Epidemiologia da insuficiência cardíaca. Rev da Sociedade de Cardiologia do Rio Grande do Sul. 2003;12:39-42. 


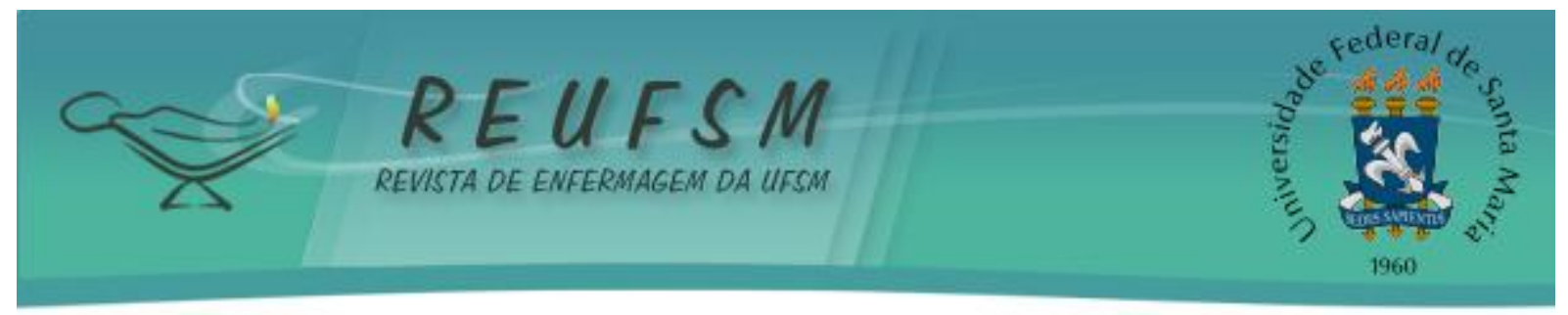

3. Araujo DV, Tavares LR, Veríssimo R, Ferraz MB, Mesquita ET. Custos da insuficiência cardíaca no Sistema Único de Saúde. Arq bras cardiol. 2005;84(5):422-7.

4. Nohria A, Tsang SW, Lewis EF, Jarcho JA, Mudge GH. Clinical assessment identifies hemodynamic profiles that predict outcomes in patients admitted with heart failure. JACC Cardiovasc Imaging. 2003;41(10):1797-804.

5. North Americam Nursing Diagosis Association. Diagnósticos de Enfermagem da NANDA: definições e classificações 2009-2011. Porto Alegre: Artmed; 2009.

6. Dougherty CM. The nursing diagnosis of decreased cardiac output. Nurs Clin North Am. 1985;20(4):787-99.

7. Calsinski CA, Barros ALBL. Nursing diagnoses in patients with a congestive heart failure. Int J Nurs Terminol Classif. 2003;14(4):1.

8. Stevenson LW, Perloff JK. The limited reliability of physical signs for estimating hemodynamics in chronic heart failure. JAMA. 1989;10(6):884-88.

9. Rohde LE, Silva LB da, Goldraich L, Grazziotin TC, Palombini DV, Polanczyk CA, et al. Reliability and prognostic value of traditional signs and symptoms in outpatients with congestive heart failure. Can J Cardiol. 2004;20(7):697-702.

10. Brandalize DL, Kalinowski CE. Processo de enfermagem: vivências na implantação da fase de diagnóstico. Cogitare enferm. 2005;10(3):53-7.

11. Cruz DALM, Pimenta CAM. Prática baseada em evidências aplicada ao raciocínio diagnóstico. Rev latinoam enferm. 2005;13(3):415-22.

12. Boery RNS de, Quatrini HCPG, Barros ALBL de. Definições operacionais das características definidoras do diagnóstico de enfermagem volume de líquidos excessivo. Acta paul enferm. 2005;18(2):197-202.

13. Grant JS, Kinney MR. The need for operational definitions for defining characteristics nursing diagnoses. Int J Nurs Terminol Classif. 1991;2(4):181-5.

14. Fehring R. Methods to validate nursing diagnoses. Heart Lung. 1987;16(6):625-9.

15. Peggy DB. Sinais e sintomas. $1^{\text {a }}$ ed. Rio de Janeiro: Guanabara Koogan; 2006.

16. Shaun C, Atwod E. Peripheral edema. Arch Intern Med. 2002;113:580-6.

17. Coelho EM. Mecanismos de formação de edemas. Medicina. 2004;37:189-98.

18. Smeltzer SC, Bare BG. Tratado de enfermagem médico-cirúrgica. $9^{\mathrm{a}}$ ed. Rio de Janeiro: Guanabara Koogan; 2002.

19. Biolo A, Netto R, Dora JM, Polanczyk CA. Exame do sistema cardiovascular. In: Barros EJG, Albuquerque GC, Pinheiro CTS, Czepielewski MA, editores. Exame clínico. Porto Alegre: Artmed; 2005. p.163-82.

20. Silver MA, Cianci P, Risano CL. Outpatient managment of heart failure-program development and experience in clinical practice. Illinois: The Heart Failure Institute and Heart Failure Center; 2004.

21. Rabelo ER, Aliti GB, Domingues FB, Ruschel KB, Brun AO. O que ensinar aos pacientes com insuficiência cardíaca e porquê: o papel dos enfermeiros em clínicas de insuficiência cardíaca. Rev latinoam enferm. 2007;15(1):165-70.

22.Martinez JAB, Pádua Al, Terra Filho J. Dispnéia. Medicina. 2004;37:199-207. 


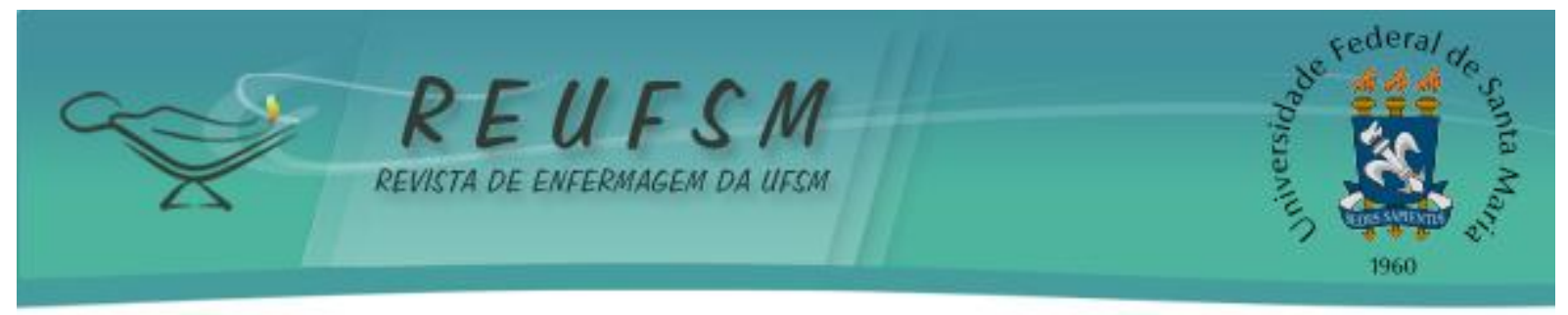

23.Shiber JR, Santana J. Dyspnea. Med Clin North Am. 2006;90:453-79.

24. Sarkar S, Amelung PJ. Evaluation of the dyspneic patient in the office. Primary care. 2006;33(3):643-57.

25. Silva ERR, Lucena AF, e colaboradores. Diagnósticos de enfermagem com base em sinais e sintomas. $1^{\text {a }}$ ed. Porto Alegre. Artmed; 2011.

26. Portilla D, Shaver MJ. Insuficiência renal aguda. In: Andreoli TE, Carpenter CCJ, Grings RC, Loscalzo J, editores. Cecil medicina interna básica. $6^{\text {a }}$ ed. Rio de Janeiro: Elsevier; 2005. p.299-308.

27. Seligman BGS, Seligmam R, Wajnberg S, Barros E. Sinais vitais. In: Barros EJG, Albuquerque GC, Pinheiro CTS, Czepielewski MA, editores. Exame clínico. Porto Alegre: Artmed; 2005.

28. Osório CMS. Semiologia psiquiátrica. In: Barros EJG, Albuquerque GC, Pinheiro CTS, Czepielewski MA, editores. Exame clínico: consulta rápida. $2^{\mathrm{a}}$ ed. Porto Alegre: Artmed; 2005. p.439-50.

29. Bickley LS. Bates' propedêutica médica. $8^{\text {a }}$ ed. Rio de Janeiro: Guanabara Koogan; 2005.

30. Tarantino AB. Sistema respiratório. In: Porto CC, editor. Semiologia médica. $5^{\mathrm{a}}$ ed. Rio de Janeiro: Guanabara Koogan; 2005. p.295-384.

31. Wender OCB, Boustany SM. Exame do sistema vascular periférico. In: Barros EJG, Albuquerque GC, Pinheiro CTS, Czepielewski MA, editores. Exame clínico. Porto Alegre: Artmed; 2005. p.289-98.

32. Mendes LA, Loscalzo J. Insuficiência cardíaca e cardiomiopatia. In: Andreoli TE, Carpenter CCJ, Griggs RC, Loscalzo JC, editores. Cecil medicina interna básica. $6^{a}$ ed. Rio de Janeiro: Elsevier; 2005.

33. Awtry EH, Loscalzo J. Estrutura e função do coração e dos vasos sanguíneos normais. In: Andreoli TE, Carpenter CCJ, Grings RC, Loscalzo J, editores. Cecil medicina interna básica. $6^{\mathrm{a}}$ ed. Rio de Janeiro: Elsevier; 2005. p.23-30.

34. Azmus AD, Maratia L. Monitorização hemodinâmica. In: Barreto SSM, Vieira SRR, Pinheiro CTS, editores. Rotinas em terapia intensiva. $3^{a}$ ed. Porto Alegre: Artmed; 2001. p.46-52.

35. Hampton JR. Descomplicando o eletrocardiograma. $5^{\mathrm{a}}$ ed. Porto Alegre: Artemd; 2000.

36. Falk K, Swedberg K, Gaston-Johansson F, Ekman I. Fatigue is a prevalent and severe symptom associated with uncertainty and sense of coherence in patients with chronic heart failure. Eur J Cardiovasc Nurs. 2007;6(2):99-104.

37. Sociedade Brasileira de Cardiologia. I Diretriz Latino Americana para avaliação e conduta na insuficiência cardíaca descompensada. Arq bras cardiol. 2004:1-132.

38. Bakos L, Bakos RM. Exame dermatológico. In: Barros E, Albuquerque GC, Pinheiro CTS, Czepielewski MA, editores. Exame clínico: consulta rápida. 2a ed. Porto Alegre: Artmed; 2005. p.79-104.

39. Barreto SSM, John BA. Sistema respiratório. In: Barros E, Albuquerque GC, Pinheiro CTS, Czepielewski MA, editores. Exame clínico: consulta rápida. $2^{\mathrm{a}}$ ed. Porto Alegre: Artmed; 2005. p.35-62.

40.Souza V, Zeitoun SS, Barros ALBL. Débito cardíaco diminuído: revisão sistemática das características definidoras. Acta paul enferm. 2011;24(1):114-9. 


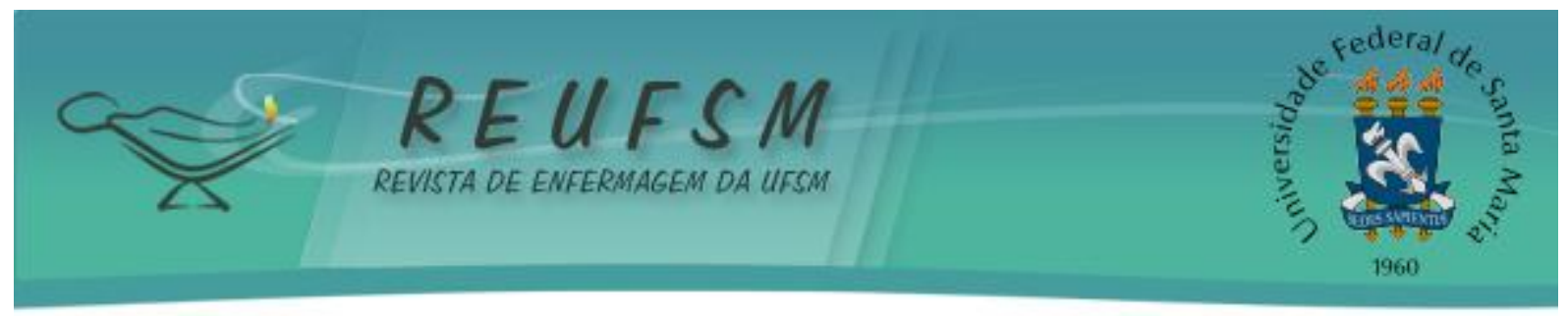

41. Lopes JL, Altino D, Silva RCG. Validação de conteúdo das atuais e de novas características definidoras do diagnóstico de enfermagem débito cardíaco diminuído. Acta paul enferm. 2010;23(6):764-8.

42. Brandão SMG, Altino DM, Silva RCG, Lopes JR. Defining characteristics of decreased cardiac output: A literature review. Int J Nurs Terminol Classif. 2011:22(2):92-102.

43. Martins QCS, Aliti G, Rabelo ER. Decreased cardiac output: clinical validation in patients with decompensated heart failure. Int J Nurs Terminol Classif. 2010;21(4):156-165.

Data de recebimento: 22/06/2011

Data de aceite: 06/11/2011

Contato com autor responsável: Quenia Camille Soares Martins

Endereço: Av. Abel Cabral 1397/1402, Nova Parnamirim, Parnamirim, RN.

CEP: $59.151-250$

E-mail: queniacamille@terra.com.br 